\title{
Estudo da diferença das temperaturas retal e do canal auditivo de acordo com a conformação da concha acústica em cães
}

\author{
Study of the difference in rectal and ear canal temperature \\ according to the conformation of the acoustic conch in dogs
}

\author{
Eduardo Negri Mueller ${ }^{1 *}$; Lucimara Konflanz Bergmann²; \\ Andreia Nobre Anciuti ${ }^{3}$; Mariana Teixeira Tillmann4; Márcia de Oliveira Nobre ${ }^{5}$
}

\section{Resumo}

\begin{abstract}
A termometria auditiva vem sendo utilizada para mensuração da temperatura em cães. Porém, existem diferentes conformações da concha acústica nesta espécie, sendo que orelhas pendulares podem alterar o microclima auditivo. Portanto, objetivou-se estudar a diferença das temperaturas retal e do canal auditivo de acordo com a conformação da concha acústica em cães. Foram utilizados 111 cães hígidos com orelhas ereta (grupo I), semipendular (grupo II) e pendular (grupo III) com 37 animais cada. A aferição da temperatura retal (TR) foi realizada com termômetro tradicional coluna de mercúrio e aferição da temperatura de ambas as orelhas externas com termômetro auricular infravermelho. Foi obtida temperatura auditiva média (TAM) e realizada diferença entre TR e TAM, sendo os resultados classificados em escores, $\mathrm{A}=\mathrm{TAM}<\mathrm{TR}$ em até $1,2^{\circ} \mathrm{C}$ e $\mathrm{B}=\mathrm{TAM}<\mathrm{TR}$ em mais de $1,2^{\circ} \mathrm{C}$. A média da TAM foi $37,15^{\circ} \mathrm{C} \pm 0,81$, enquanto que a da TR foi $38,68^{\circ} \mathrm{C} \pm 0,59$. A diferença média entre TR e TAM foi $1,53^{\circ} \mathrm{C} \pm 0,75$. Setenta e uma orelhas $(63,96 \%)$ foram classificadas no escore B e $40(36,04 \%)$ no escore A. A média da TAM foi $37,17^{\circ} \mathrm{C} \pm 0,85,37,35^{\circ} \mathrm{C} \pm 0,50$ e $36,93^{\circ} \mathrm{C} \pm 0,96$ respectivamente nos grupos I, II e III. Não houve influência do tipo de orelha na TAM $(\mathrm{p}=0,5097)$. A diferença média entre a TR e TAM no grupo I foi $1,57^{\circ} \mathrm{C} \pm 0,65$, no grupo II $1,26^{\circ} \mathrm{C} \pm 0,49$ e no III $1,76^{\circ} \mathrm{C} \pm 0,96$. Predominaram orelhas no escore $\mathrm{B}$ independente do tipo $(\mathrm{p}=0,7606)$. Nas condições deste estudo a temperatura auditiva média é menor que a retal $1,53^{\circ} \mathrm{C} \pm 0,75$ e a conformação da orelha não influencia a temperatura auditiva média em cães. Palavras-chave: Orelha externa, microclima, termometria
\end{abstract}

\begin{abstract}
The auditory thermometry has being used for measurement of temperature of dogs. However there is different acoustic conch conformation in this species, being that the pendulous ears can alter the auditory microclimate. This research aimed to study the difference of rectal and auditory canal temperatures according to acoustic conch conformation in dogs. One hundred and eleven dogs were used with erect ears (group I), semi-pendulous (group II) and pendulous (group II) with 37 animals each group. The measurement of rectal temperature (TR) was realized with traditional thermometer mercury column and measurement of both external ears temperature with infrared ear thermometer. The average hearing temperature (TAM) was obtained and realized difference between TR and TAM, being the results classify in scores, $\mathrm{A}=\mathrm{TAM}<\mathrm{TR}$ until $1.2^{\circ} \mathrm{C}$ and $\mathrm{B}=\mathrm{TAM}<\mathrm{TR}$ more than $1.2^{\circ} \mathrm{C}$. The average of TAM
\end{abstract}

\footnotetext{
${ }^{1}$ Bolsista PNPD Institucional CAPES. Universidade Federal de Pelotas, UFPel, Pelotas, RS. E-mail: enmuellervet@yahoo.com.br

${ }^{2}$ Residente em Clínica Cirúrgica de Animais de Companhia, Hospital de Clínicas Veterinária. UFPel, Pelotas, RS. E-mail: lk.bergmann@gmail.com

${ }^{3}$ Discente do curso de Medicina Veterinária, UFPel, Pelotas, RS. E-mail: andreianciuti@hotmail.com

${ }^{4}$ Doutoranda, PPG em Veterinária, UFPel, Pelotas, RS. E-mail: marianatillmann@yahoo.com.br

${ }^{5}$ Prof ${ }^{a}$, Faculdade de Veterinária, UFPel, Pelotas, RS. E-mail: marciaonobre@gmail.com

* Autor para correspondência
} 
was $37.15^{\circ} \mathrm{C} \pm 0.81$, whereas that $\mathrm{TR}$ was $38.68^{\circ} \mathrm{C} \pm 0.59$. The average difference between TR and TAM was $1.53^{\circ} \mathrm{C} \pm 0.75$. Seventy one ears $(63.96 \%)$ were classify in the score $\mathrm{B}$ and $40(36.04 \%)$ in the score A. The TAM average was $37.17^{\circ} \mathrm{C} \pm 0.85,37.35^{\circ} \mathrm{C} \pm 0.50$ and $36.93^{\circ} \mathrm{C} \pm 0.96$ respectively in groups $\mathrm{I}$, II, III. There was no difference in the type of ear in TAM $(\mathrm{p}=0.5097)$. The average difference between the TR and TAM in the group I was $1.57^{\circ} \mathrm{C} \pm 0.65$, in group II $1.26^{\circ} \mathrm{C} \pm 0.49$ and in the III $1.76^{\circ} \mathrm{C} \pm 0.96$. Predominated ears in the score $\mathrm{B}$ independent of type $(\mathrm{p}=0.7606)$. In the conditions of this study the average ear temperature is lower than the rectal temperature by $1.53^{\circ} \mathrm{C} \pm 0.75$ and the conformation of the ear does not influence the average ear temperature.

Key words: External ear, microclimate, thermometry

\section{Introdução}

A avaliação da temperatura auditiva é um método alternativo para obtenção da temperatura corporal de pequenos animais, devido a sua praticidade e rapidez quando comparada a aferição por via retal (ALENCAR-JÚNIOR et al., 2007).

Este parâmetro clínico é utilizado com bons resultados em humanos (MACHADO; ANDRADE, 2008; VERTEDOR-HURTADO et al., 2009), porém em cães alguns fatores podem influenciar o microclima, e incluem anatomia da orelha, diferentes conformações da concha acústica e, características individuais como a presença de pêlos, produção de cerúmen e reações inflamatórias (WHITE, 1999; MURPHY, 2001; HARVEY; HARARI; DELAUCHE, 2004; ALENCAR-JÚNIOR et al., 2007).

Sendo assim, é necessário estudar a temperatura auditiva de cães e estabelecer critérios para a utilização deste recurso, principalmente em situações em que ocorre o impedimento ou a dificuldade da obtenção da temperatura retal, provocados principalmente por enfermidades anais e perianais. Neste contexto, objetivou-se estudar a diferença das temperaturas retal e do canal auditivo de acordo com a conformação da concha acústica em cães.

\section{Material e Métodos}

Foram avaliados 111 cães sem sinais clínicos de otite externa, desta forma totalizando 222 conchas acústicas. Os cães estudados foram 52 machos e 59 fêmeas, todos adultos.
Os animais foram mantidos em repouso durante 30 minutos que antecederam as aferições, as quais foram realizadas pelo mesmo avaliador. Inicialmente, foi obtida a temperatura retal (TR) aferida com termômetro tradicional coluna de mercúrio (Incoterm ${ }^{\circledR}$ Termômetro Clínico, modelo $\mathrm{P} 300$, precisão $-0,15+0,1^{\circ} \mathrm{C}$ ) na ampola retal durante três minutos. A temperatura do conduto auditivo externo foi avaliada primeiramente na orelha direita e imediatamente após na orelha contralateral (esquerda), obtendo-se a temperatura auditiva média (TAM). Para obtenção da temperatura do conduto auditivo externo a orelha foi suavemente tracionada caudalmente e o termômetro introduzido na porção vertical do canal auditivo externo, sendo utilizado termômetro auricular infravermelho (Omron ${ }^{\circledR}$ Mini Instant Ear Thermometer, modelo MC-510NT, precisão $\pm 0,2^{\circ} \mathrm{C}$ ), acionado e mantido até emissão de sinal sonoro. $\mathrm{O}$ tempo de avaliação decorrido entre a primeira e a última aferição foi de aproximadamente 210 segundos.

Foi realizada a diferença entre TR e TAM, sendo os resultados obtidos expressos de acordo com os seguintes escores: $\mathrm{A}=$ quando a TAM era menor que a TR $(\mathrm{TAM}<\mathrm{TR})$ em até $1,2^{\circ} \mathrm{C} ; \mathrm{B}=$ quando TAM era menor que TR (TAM $<$ TR) em mais de $1,2^{\circ} \mathrm{C}$. Para determinação dos escores foi utilizado $1,2^{\circ} \mathrm{C}$ descrito como diferença máxima entre temperaturas retal e auditiva em orelhas hígidas de cães (GRONO, 1970).

Foi obtida a média da TAM de 111 cães ( 37 em cada grupo) de acordo com a conformação da concha acústica, ereta (Grupo I), semipendular (Grupo II) ou pendular (Grupo III), e ainda foi obtida a 
média da diferença entre TR e TAM (Statistix 9.0). A análise estatística foi realizada por teste de Quiquadrado extraído do programa Statistix 9.0.

\section{Resultados e Discussão}

O valor médio da TAM das orelhas eretas (Grupo I) foi de $37,17^{\circ} \mathrm{C} \pm 0,85$, das orelhas semipendulares (Grupo II) de $37,35^{\circ} \mathrm{C} \pm 0,50$ e das orelhas pendulares foi de $36,93^{\circ} \mathrm{C} \pm 0,96$, não havendo diferenças entre o tipo de orelha e a TAM $(p=0,5097)$. O estudo de Huang e Huang (1999), também demonstrou temperatura auditiva próxima de $38,0^{\circ} \mathrm{C}$ sem diferenças significativas entre os tipos de orelha. A diferença entre a TR e TAM nas orelhas eretas foi de $1,57^{\circ} \mathrm{C} \pm 0,65$ enquanto que nas semipendulares foi de $1,26^{\circ} \mathrm{C} \pm 0,49$ e nas pendulares de $1,76^{\circ} \mathrm{C} \pm 0,96$, não tendo sido demonstrado diferença estatística entre os grupos $(\mathrm{p}=0,7606)$ (Tabela 1$)$.

A TAM variou de $34,35^{\circ} \mathrm{C}$ a $39,25^{\circ} \mathrm{C}$ (média $37,15^{\circ} \mathrm{C} \pm 0,81$ ), enquanto que a TR variou de $36,8^{\circ} \mathrm{C}$ a $41,0^{\circ} \mathrm{C}$ (média $38,68^{\circ} \mathrm{C} \pm 0,59$ ). A diferença média entre TR e TAM foi de $1,53^{\circ} \mathrm{C} \pm 0,75$, sendo que nos cães machos esta diferença foi de $1,50^{\circ} \mathrm{C} \pm 0,76 \mathrm{e}$ nas fêmeas de $1,55^{\circ} \mathrm{C} \pm 0,74$. A diferença foi maior do que a descrita na literatura que considera a temperatura auditiva em orelhas hígidas como sendo em média $0,6^{\circ} \mathrm{C}$ menor que a retal, variando de $0,1^{\circ} \mathrm{C}$ a $1,2^{\circ} \mathrm{C}$ (GRONO, 1970). Ao agrupar os valores obtidos em escores foi observado que 71 cães $(63,96 \%)$ apresentaram a TAM menor que a TR (TAM $<$ TR) em mais de $1,2^{\circ} \mathrm{C}$ (escore B) e 40 $(36,04 \%)$ apresentaram a TAM menor que a TR $(\mathrm{TAM}<\mathrm{TR})$ em até $1,2^{\circ} \mathrm{C}$ (escore $\left.\mathrm{A}\right)$.

Tabela 1. Escore da diferença das temperaturas retal e auditiva média de acordo com a conformação da concha acústica hígida de cães. Pelotas-RS, 2011.

\begin{tabular}{lccc}
\hline & \multicolumn{3}{c}{ Escore* } \\
\cline { 2 - 4 } Conformação da concha acústica & $\mathbf{A}$ & $\mathbf{B}$ & Total \\
& $\mathbf{n}(\mathbf{\%})$ & $24(\mathbf{\%})$ & $\mathbf{n}(\mathbf{\%})$ \\
\hline Ereta & $13(11,71)$ & $22(1,62)$ & $\mathbf{3 7 ( 3 3 , 3 )}$ \\
Semipendular & $15(13,51)$ & $\mathbf{3 7}(\mathbf{3 3 , 3})$ \\
Pendular & $12(10,81)$ & $25(22,52)$ & $\mathbf{3 7}(\mathbf{3 3 , 3})$ \\
\hline Total & $40(36,04)$ & $71(63,96)$ & $111(100,0)$ \\
\hline
\end{tabular}

$* \mathrm{~A}=$ temperatura auditiva média menor que a retal $(\mathrm{TAM}<\mathrm{TR})$ em até $1,2^{\circ} \mathrm{C} ; \mathrm{B}=$ temperatura auditiva média menor que a retal (TAM<TR) em mais de $1,2^{\circ} \mathrm{C}$.

Fonte: Elaboração dos autores.

Estudo comparando a temperatura auditiva média das orelhas direita e esquerda com a temperatura retal observou respectivamente as seguintes médias $37,20^{\circ} \mathrm{C} \pm 0,89, \quad 37,29^{\circ} \mathrm{C} \pm 0,89$ e $37,41^{\circ} \mathrm{C} \pm 1,15$, demonstrando uma alta correlação entre a TAM e a TR (WIEDEMANN et al., 2006). Semelhante avaliação foi realizada obtendo média de $38,4^{\circ} \mathrm{C} \pm 0,7$ na orelha direita, esquerda e temperatura retal de $39,1^{\circ} \mathrm{C} \pm 0,6$ (HUANG; HUANG, 1999).

Foram avaliadas apenas orelhas hígidas, porque o processo inflamatório na orelha externa pode alterar o microclima auditivo (NASCENTE et al., 2006;
MUELLER et al., 2011a; 2011b) e, possivelmente, altera a relação desta temperatura com a temperatura retal. Além disto, já foi encontrada alta correlação entre as temperaturas retal e auditiva de cães com otite externa leve ou severa (GONZÁLEZ et al., 2002). Também é necessário considerar que nos casos de otite externa a alta sensibilidade a dor, devido a inflamação pode dificultar o uso da termometria timpânica em cães (WIEDEMANN et al., 2006).

De acordo com os resultados deste estudo, o termômetro auditivo não serve de parâmetro 
clínico para obtenção da temperatura sistêmica, não substituindo o uso de termômetro por via retal em cães. Embora seja importante utilizar este método em pediatria e em condições em que haja impedimento da aferição da temperatura retal, considerando que é uma técnica rápida, prática e não invasiva.

\section{Conclusão}

Nas condições deste estudo a temperatura auditiva média é menor que a retal $1,53^{\circ} \mathrm{C} \pm 0,75$ e a conformação da orelha não influencia a temperatura auditiva média em cães.

\section{Agradecimentos}

Ao CNPq pelo apoio financeiro e a CAPES pela concessão da bolsa de pós-graduação. Beneficiário de auxílio financeiro da CAPES - Brasil.

\section{Referências}

ALENCAR-JÚNIOR, V. P.; AQUINO, M.; CARARETO, R.; SOUSA, M. G. Avaliação do termômetro auricular em cães normotérmicos. Acta Scientiae Veterinariae, Porto Alegre, v. 35, p. 408-409, 2007. Suplemento 2.

GONZÁLEZ, A. M.; MANN, F. A.; PREZIOSI, D. E.; MEADOWS, R. L.; WAGNER-MANN, C. C. Measurement of body temperature by use of auricular thermometers versus rectal thermometers in dogs with otitis externa. Journal of the American Veterinary Medical Association, Schaumburg, v. 221, n. 3, p. 378380, 2002. Disponível em: <http://www.admon.com/wpcontent/uploads/2010/09/JAVMA-Article.pdf>. Acesso em: 01 ago. 2011.

GRONO, L. R. Studies of the microclimate of the external auditory canal in the dog I - aural temperature. Research in Veterinary Science, Londres, v. 2, p. 307-311, 1970.

HARVEY, R. G.; HARARI, J.; DELAUCHE, A. J. Doenças do ouvido de cães e gatos. Rio de Janeiro: REVINTER, 2004. 272 p.

HUANG, H. P.; HUANG, H. M. Effects of ear type, sex, age, body weight, and climate on temperatures in the external acoustic meatus of dogs. American Journal of Veterinary Research, Schaumburg, v. 60, n. 9, p. 11731176, 1999.
MACHADO, A. V. S.; ANDRADE, M. M. M. O ciclo vigília/sono antes e após intervenção na rotina de idosos institucionalizados. Revista Brasileira de Ciências do Envelhecimento Humano, Passo Fundo, v. 5, n. 2, p. 3345, 2008. Disponível em: <http://www.upf.br/seer/index. php/rbceh/article/view/127>. Acesso em: 20 jul. 2011.

MUELLER, E. N.; GUIOT, Ê. G.; TILLMANN, M. T.; FELIX, A. O. C.; PEREIRA, I. C.; SCHRAMM, R. C.; NOBRE, M. O. Avaliação do efeito da lavagem do canal auditivo externo em orelhas com otite externa purulenta bilateral. MEDVEP - Revista Científica de Medicina Veterinária-Pequenos Animais e Animais de Estimação, Curitiba, v. 9, n. 28, p. 147-150, 2011a.

MUELLER, E. N.; GUIOT, Ê. G.; WILHELM, G.; FERNANDES, C. P. M.; SCHUCH, L. F. D.; NOBRE, M. O. Otite externa ceruminosa e purelenta em cães. $A$ Hora Veterinária, Porto Alegre, v. 30, n. 179, p. 38-40, $2011 b$.

MURPHY, K. M. A review of techniques for the investigation of otitis externa and otitis media. Clinical Techniques in Small Animal Practice, Philadelphia, v. 16, p. 236-241, 2001. Disponível em: $<$ http://www.sciencedirect.com/science/article/pii/ S1096286701800426>. Acesso em: 15 jul. 2011.

NASCENTE, P. S.; CLEFF, M. B.; ROSA, C. S.; SANTOS, D. V.; MEIRELES, M. C. A.; MELLO, J. R. B. Otite externa em pequenos animais: uma revisão. MEDVEP - Revista Científica de Medicina Veterinária Pequenos Animais e Animais de Estimação, Curitiba, v. 4, n. 11, p. 52-59, 2006.

VERTEDOR-HURTADO, M. V.; PADÍN-LÓPEZ, S.; CARREIRA-PASTOR, M. J.; LÓPEZ-MARTÍNEZ, J. M. Termometría timpánica en pediatría como alternativa al termómetro de mercurio. Enfermería Clínica, Barcelona, v. 19, n. 3, p. 115-120, 2009. Disponível em: <http://www.sciencedirect.com/ science/article/pii/ S1130862109000485>. Acesso em: 15 jul. 2011.

WHITE, P. D. Medical management of chronic otitis in dogs. Compendium on Continuing Education for the Practicing Veterinarian, Trenton, v. 21, n. 8, p. 716-727, 1999.

WIEDEMANN, G. G. S.; SCALON, M. C.; PALUDO, G.; SILVA, I. O.; BOERE, V. Comparison between tympanic and anal temperature with a clinical infrared ray thermometer in dogs. Arquivo Brasileiro de Medicina Veterinária e Zootecnia, Belo Horizonte, v. 58, n. 4, p. 503505, 2006. Disponível em: <http://www.scielo.br/scielo. php?pid=S0102-09352006000400008\& script $=$ sci arttext>. Acesso em: 15 jul. 2011. 